\title{
Existence of Random Attractors for Nonlinear Plate Equations with Thermal Memory Effect and Additive Noise
}

Xia Li, Xianyun $\mathrm{Du}^{*}$

College of Applied Mathematics, Chengdu University of Information Technology, China

DOI: $10.36347 /$ sipms.2020.v07i08.006

| Received: 18.08.2020 | Accepted: 26.08.2020 | Published: 30.08 .2020

*Corresponding author: Xianyun Du

Abstract

Review Article

In this paper, the long-time dynamics of the nonlinear plate equations with thermal memory effect and additive noise caused by non-Fourier heat flow law is studied. We study the existence of random attractors for stochastic dynamical systems related to equations.

Keywords: Nonlinear plate equations, thermal memory, stochastic dynamic system, random attractor, additive noise. Copyright @ 2020: This is an open-access article distributed under the terms of the Creative Commons Attribution license which permits unrestricted use, distribution, and reproduction in any medium for non-commercial use (NonCommercial, or CC-BY-NC) provided the original author and source are credited.

\section{INTRODUCTION}

In this paper, the research methods in the literature [4-6] consider the nonlinear Plate equation with thermal memory effect caused by the non-Fourier heat flow law with additive noise and has a random attractor in the stochastic dynamic system. The study equation is as follows

$$
\left\{\begin{array}{c}
\theta_{\mathrm{t}}-\Delta u_{t}+\int_{0}^{\infty} k(s)[-\Delta \theta(t-s)] d s=\sum_{j=1}^{m} \phi_{j} d w_{j} \quad t \in R^{+}, x \in U \\
u_{t t}-\Delta u_{t}+\Delta(\Delta u+\theta)+f(u)=0
\end{array}\right.
$$

Boundary condition

$$
\begin{array}{ll}
u(t)=\Delta u(t)=0 & t \geq 0, x \in \Gamma \\
\theta(t)=0 & t \in R, x \in \Gamma
\end{array}
$$

Initial value condition

$$
\begin{array}{ll}
u(0)=u_{0} & x \in U \\
u_{t}(0)=v_{0} & x \in U \\
\theta(0)=\theta_{0} & x \in U \\
\theta(-s)=\Psi(s) & (x, s) \in U \times R^{+}
\end{array}
$$

Among them, $\sum_{j=1}^{m} \phi_{j} d w_{j}=\sum_{j=1}^{m} \phi^{j} w^{j} d t . U$ is an appropriate smooth boundary $\Gamma$ in $R^{2}, u=u(t, x)$ is a real-valued function on $U \times[0,+\infty), \theta$ is the temperature change of the self-balanced reference value, $u$ is the vertical displacement of the plate, and temperature, assuming the memory kernel function $k: R^{+} \mapsto R$.It is a positive bounded convex function and disappears at infinity. For random terms $\phi_{j}(x) \in H_{0}^{2}(U) \cap H^{3}(U)(j=1,2, \cdots, m),\left\{\omega_{j}\right\}_{j=1}^{m}$ is an independent bilateral real-valued Wiener process on probability space $(\Omega, F, P)$. Among them, function $\Psi: R^{+} \times U \mapsto R$ is called the initial history of 


$$
\Omega=\left\{\omega=\left(\omega_{1}, \omega_{2}, \cdots, \omega_{m}\right) \in C\left(R, R^{m}\right): \omega(0)=0\right\} .
$$

Given a tight open topology, F is a P-complete Borel $\sigma$-algebra on $\Omega$.

There is a lot of research work on the thermoelastic plate equation in the literature. The Plate equation is derived from the elastic vibration equation established by Woinowsky-Krieger ([12]) and Berger ([13]). For the Plate equation, the latest study of the attenuation estimation and asymptotic curve of the Plate equation with memory (cf[20]); in [23], the quasistability properties of the system are established, and the existence of global attractors and exponential attractors with finite fractal dimensions is obtained; studied the precise controllability of the thermoelastic plate equation with memory (cf[21]);obtained the general stability results of the viscoelastic plate equation with past history and general kernel (cf[22]), etc. In these studies, we have noticed that in recent decades, the long-term behavior of the deterministic Plate equation or the solution of a single Plate equation has been studied by many scholars. Such as, the author of the literature [14] in 2005 considered the following evolution problem in the two-dimensional theory of linear genetic thermoelasticity,

$$
\left\{\begin{array}{c}
u_{t t}(t)+\Delta(\Delta u(t)+\vartheta(t))=0 \\
\vartheta_{t}(t)+\int_{0}^{\infty} k(s)[c \vartheta(t-s)-\Delta \vartheta(t-s)] d s-\Delta u_{t}(t)=0
\end{array}\right.
$$

which simulates Kirchhoff thermoelastic thin plate with heat conduction memory effect. The exponential stability of the solution of the equation is considered.

The existence and uniqueness of the global solution and the existence of the global attractor are proved.

However, in real life, many systems will inevitably be affected by random factors. Therefore, this paper studies the existence of random attractors of stochastic Plate equations in stochastic dynamic systems based on [7]. This article is organized as
In 2008, a nonlinear Plate equation with thermal memory effect was considered in [7].

$$
\left\{\begin{array}{c}
\vartheta_{t}-\Delta u_{t}+\int_{0}^{\infty} k(s)[-\Delta \vartheta(t-s)] d s=0 \\
u_{t t}-\Delta u_{t}+\Delta(\Delta u+\vartheta)+f(u)=0
\end{array}\right.
$$

follows. In the next section, we will review some basic concepts and properties of general stochastic dynamic systems. In Section 3, we provide some basic settings related to formula (1.1) and show that it generates a stochastic dynamic system in the appropriate function space. Section 4 is devoted to proving the existence of the unique random attractor of the stochastic dynamic system.

\section{Basic Definition TheOREM}

Definition 2.1 ([8]) The random dynamic system $\left(\Omega, F, P,\left(\vartheta_{\mathrm{t}}\right)_{t \in R}\right)$ is called $\operatorname{RDS} \varphi$.

$$
\begin{gathered}
\varphi: \quad T \times X \times \Omega \rightarrow X \\
(t, x, \omega) \mapsto \varphi(t, \omega) x,
\end{gathered}
$$

where identity map $\varphi(0, \omega)=i d$ on $X$,and has the following relationship

$$
\varphi(t+s, \omega)=\varphi\left(t, \vartheta_{s} \omega\right) \circ \varphi(s, \omega),
$$

among them $t, s \in T, \omega \in \Omega$, ० represent combinations. A cluster of maps $\varphi(t, \omega)$ satisfying (2.1) is called a cocycle, and (2.1) is also the nature of cocycle. The inverse of $\varphi(t, \omega)$ is $\varphi(t, \omega)^{-1}=\varphi\left(-t, \vartheta_{t} \omega\right)$.

Definition 2.2 ([8]) Random set A attracts another random set B .if

$$
d\left(\varphi\left(t, \vartheta_{-t} \omega\right) B\left(\vartheta_{-t} \omega\right), A(\omega)\right) \underset{n \rightarrow \infty}{\longrightarrow} 0,
$$

then call $\mathrm{A}$ as an attractive set.

Definition 2.3 ([8]) Random set $\mathrm{K}$ attracts another random set $\mathrm{B}$, if $t_{B}(\omega)$ exists, for all $\mathrm{t} \geq t_{B}(\omega)$ there is

$$
\varphi\left(t, \vartheta_{-t} \omega\right) B\left(\vartheta_{-t} \omega\right) \subset K(\omega),
$$

$t_{B}(\omega)$ is called absorption time. Then $\mathrm{K}$ is called absorption set. 
Theorem 2.1 ([8] [9]) Let $\Phi$ be the measurable power system $\left(\Omega, F, P,\left(\vartheta_{\mathrm{t}}\right)_{t \in R}\right)$ and a continuous random power system in space $\mathrm{X}$. If there is a random compact set $K(\omega)$, such that for any nonrandom bounded set $B \subset X$, there is

$$
\lim d\left(\Phi\left(t, \vartheta_{-t} \omega\right) B, K(\omega)\right)=0, t \rightarrow+\infty
$$

then $\Phi$ has a random attractor

$$
\mathrm{A}=\{A(\omega)\}_{\omega \in \Omega}=\overline{\bigcup_{B \subset X} \Lambda_{B}(\omega)},
$$

where B is taken through the bounded subset of $\mathrm{X}, \Lambda_{B}(\omega)$ is the $\omega$-limit set of $\mathrm{B}$, ie

$$
\Lambda_{B}(\omega)=\bigcap_{s \geq 0} \overline{\bigcup_{t \geq s} \Phi\left(t, \vartheta_{-t} \omega\right) B} .
$$

Theorem 2.2 ([15] ) Suppose $X_{0}, X_{1}, X$ are three Banach spaces, of which $X_{0}, X$ are reflexive and satisfy

$$
X_{0} \rightarrow X \rightarrow X_{1} \text { (Embed), }
$$

here embedding $X_{0} \rightarrow X$ is tight. If $Y \subset L_{\mu}^{2}\left(R^{+}, X\right)$ meets the following conditions:

(i) $\quad \mathrm{Y}$ is bounded in $L_{\mu}^{2}\left(R^{+}, X_{0}\right) \cap H_{\mu}^{1}\left(R^{+}, X_{1}\right)$;

(ii) $\quad$ For $\forall s \in R^{+}$, there is $K_{0}>0$, so that $\sup _{\eta \in Y}\|\eta(s)\|_{X}^{2} \leq K_{0}$;

then $\mathrm{Y}$ is relatively tight in $L_{\mu}^{2}\left(R^{+}, X\right)$.

Lemma 2.1 (19]) Let $X$ be a Banach space and $Z \in C([0, \infty), X)$. Let $E: X \rightarrow R$ be a function bounded from below such that $E(Z(0)) \leq M$ for $Z \in X$.If

$$
\frac{d}{d t} E(Z(t))+\sigma\|Z(t)\|_{X}^{2} \leq \chi,
$$

for some $\sigma \geq 0, \chi \geq 0$ independent of $\mathrm{Z}$, then for all $\varepsilon_{1}>0, t_{0}=t_{0}\left(M, \varepsilon_{1}\right)>0$, such that

$$
E(Z(t)) \leq \sup _{\xi \in X}\left\{E(\xi): \sigma\|\xi\|_{X}^{2} \leq \chi+\varepsilon_{1}\right\}, \forall t \geq t_{0} .
$$

Lemma 2.2 For $m<\varepsilon<l, g=0,1, \forall B_{i} \in V^{0}, i=1,2$, then

(1) $-\varepsilon\left(A u, B_{1}\right) \geq-\frac{\alpha \varepsilon}{4}\|u\|_{2}^{2}-\frac{\varepsilon}{\alpha}\left\|B_{1}\right\|^{2}$,

(2) $-\int_{U} B_{1} A u d x \geq-\frac{\alpha}{4}\left\|B_{1}\right\|^{2}-\frac{1}{\alpha}\|u\|_{2}^{2}$,

(3) $\left| \pm\left(A^{g} B_{1}, B_{2}\right)\right| \leq\left\|A^{g} B_{1}\right\|\left\|B_{2}\right\| \leq \frac{1}{\alpha}\left\|A^{g} B_{1}\right\|^{2}+\frac{\alpha}{4}\left\|B_{2}\right\|^{2}$,

(4) $\left| \pm \int_{0}^{\infty} \varepsilon \mu(s)<A^{g} B_{1}, \eta^{t}(s)>d s\right| \leq \frac{\varepsilon \alpha k_{0}}{4}\left\|A^{g / 2} B_{1}\right\|^{2}+\frac{\varepsilon}{\alpha D_{1}^{g / 2}}\left\|\eta^{t}\right\|_{M^{1}}^{2}$.

\section{BASIC SETTINGS}

Assume that the memory kernel function $\mu(s)$ and the nonlinear term $f(u)$ satisfy the following conditions:

(1) For any $s \in R^{+}$, there is $\delta>\frac{23 D_{1}+3}{6\left(D_{1}-2\right)}>0$, such that

$$
\mu(s) \in C^{1}\left(R^{+}\right) \bigcap L^{1}\left(R^{+}\right), \mu(s) \geq 0, \mu^{\prime}(s)+\delta \mu(s) \leq 0 .
$$


(2) Assuming that $\mathrm{k}$ is zero at infinity, in addition, define $\mu(s)=-k^{\prime}(s)$.In recent years, various development equations under the non-Fourier heat flow law have aroused the interest of many

$$
q(t)=-\int_{0}^{\infty} k(s) \nabla \theta(t-s) d s,
$$

where $\mathrm{k}$ is the thermal conductivity relaxation kernel, and the existence of the convolution term in the above formula means the finite propagation velocity of thermal disturbance, so in this case, the corresponding

equation is hyperbolic. It is easy to see that if $\mathrm{k}$ is the Dirac mass at zero, the above formula can be simplified to the classic Fourier's law $\mathrm{q}=-\nabla \theta$. In addition,

$$
k(s)=\frac{1}{\sigma} e^{-\frac{s}{\sigma}}, \sigma>0
$$

among them, $\frac{2}{3}<k_{0}<\min \left\{4, \frac{\delta}{2}-\frac{\delta}{D_{1}}-\frac{5}{4}-\frac{1}{4 D_{1}}, 8-\frac{10}{D_{1}}\right\}$.

(within the limited range of each parameter, $\frac{\delta}{2}-\frac{\delta}{D_{1}}-\frac{5}{4}-\frac{1}{4 D_{1}}$ and $8-\frac{10}{D_{1}}$ are less than $\frac{2}{3}$ ).

(3) The nonlinear function $f(u)$ satisfies:

$$
\begin{gathered}
f(0)=0,\left|f^{\prime}(s)\right| \leq C_{0}(1+|s|), \forall s \in R, \\
F(s)=\int_{0}^{s} f(r) d r \geq C_{1}\left(|s|^{3}-1\right), \forall s \in R, \\
\liminf _{|s| \rightarrow \infty} \frac{s f(s)-C_{2} F(s)}{s^{2}} \geq 0, \forall s \in R . \\
\liminf _{|s| \rightarrow+\infty} \frac{f(s)}{s}>-\frac{1}{C_{U}} .
\end{gathered}
$$

among them, $0<C_{2} \leq \frac{2 C_{0} u^{2}+C_{0}|u|^{3}}{2 C_{1}\left(|u|^{3}-1\right)}, C_{i}>0(i=0,1, \cdots)$ is a normal number, in this article the constant $\mathrm{C}$ changes, each row or even the same row of $\mathrm{C}$ is not equal. $C_{U}$ is the best constant that depends only on $U$, then

$$
\|u\|_{L^{2}(U)}^{2} \leq C_{U}\|\mathrm{~A} u\|_{L^{2}(U)}^{2}
$$

Consider the positive operator A on $L^{2}(U)$, defined as $\mathrm{A}=-\Delta=\nabla^{2}$ and bounded by $D(\mathrm{~A})=H^{2}(U) \cap H_{0}^{1}(U)$. For $r \in \mathbf{R}$, consider Hilbert space $v^{r}=D\left(\mathrm{~A}^{r / 2}\right)$, define the inner product

$$
\begin{gathered}
\left(u_{1}, u_{2}\right)_{v^{r}}=\left(\mathrm{A}^{r / 2} u_{1}, \mathrm{~A}^{r / 2} u_{2}\right), \forall u_{1}, u_{2} \in v^{r}, \\
\|u\|_{r}^{2}=\left(A^{r / 2} u, A^{r / 2} u\right), u \in v^{r}, \\
\|u\|^{2}=(u, u), u \in v^{0},
\end{gathered}
$$

where $(\cdot, \cdot)$ and $\|\cdot\|$ represent the inner product and norm in $v^{0}=L^{2}(U)$. Use $((\cdot, \cdot)),\|\cdot\|_{2}$ to denote the inner product and norm of $v^{2}=H_{0}^{2}(U)$, respectively. For $r_{1}>r_{2}$, embedding $v^{r_{1}} \rightarrow v^{r_{2}}$ is tight.

We introduce a weighted Hilbert memory space $\mathrm{M}^{1+r}=L_{\mu}^{2}\left(R^{+} ; v^{r}\right)$, of which $r \in R$. Define the inner product as follows

$$
\begin{gathered}
\left\langle\eta_{1}, \eta_{2}\right\rangle_{\mathrm{M}^{r}}=\int_{0}^{\infty} \mu(s)\left\langle\mathrm{A}^{r / 2} \eta_{1}(s), \mathrm{A}^{r / 2} \eta_{2}(s)\right\rangle d s . \forall \eta_{1}, \eta_{2} \in \mathrm{M}^{1+r}, \\
\|\eta\|_{\mathrm{M}^{1+r}}^{2}=\int_{0}^{\infty} \mu(s)\|\eta(s)\|_{2}^{2} d s, \forall \eta(s) \in \mathrm{M}^{1+r} \\
\|\eta\|_{\mathrm{M}^{1}}^{2}=\int_{0}^{\infty} \mu(s)\|\eta(s)\|_{\mathrm{M}^{1}}^{2} d s, \forall \eta(s) \in \mathrm{M}^{1}
\end{gathered}
$$


Here we notice that for $r_{1}>r_{2}$, embedding $\mathrm{M}^{r_{1}} \rightarrow \mathrm{M}^{r_{2}}$ are continuous but not compact (cf.[17,18]).

Finally, we define the inner product Hilbert space

$$
V^{r}=v^{2+r} \times v^{r} \times v^{r} \times \mathrm{M}^{1+r}, r \in R .
$$

For all $z=\left(z_{1}, z_{2}, z_{3}, z_{4}\right)^{T} \in V^{r}$, there is norm

$$
\|z\|_{V^{r}}^{2}=\left\|\mathrm{A}^{(2+r) / 2} z_{1}\right\|^{2}+\left\|\mathrm{A}^{r / 2} z_{2}\right\|^{2}+\left\|\mathrm{A}^{r / 2} z_{3}\right\|^{2}+\left\|z_{4}\right\|_{\mathrm{M}^{1+r}}^{2},
$$

when $\mathrm{r}=0$, there is $V^{0}=v^{2} \times v^{0} \times v^{0} \times \mathrm{M}^{1}$.

\section{The Existence of RAndom AtTractors}

It is convenient to work in the history space setting by introducing the so-called summed past history of $\theta$ which is defined as follows (cf.[7])

$$
\eta^{t}(s)=\int_{0}^{s} \theta(t-y) d y, \quad(t, s) \in[0, \infty) \times R^{+},
$$

the variable $\eta^{t}$ (formally) satisfies the linear equation

$$
\eta_{t}^{t}(s)+\eta_{s}^{t}(s)=\theta(t), \text { in } \Omega, \quad(t, s) \in R^{+} \times R^{+},
$$

boundaries and initial conditions

$$
\begin{gathered}
\eta^{t}(0)=0, \text { in } \Omega, t \geq 0, \\
\eta^{0}(s)=\eta_{0}(s)=\int_{0}^{s} \psi(y) d y, \text { in } \Omega, s \in R^{+} .
\end{gathered}
$$

the linear operator $\mathrm{T}$ introduced in $\mathrm{M}^{1}$ is defined as

$$
T \eta=-\eta_{s}, \eta \in D(T)
$$

its boundary is

$$
D(T)=\left\{\eta \in \mathrm{M}^{1} \mid \eta_{s} \in \mathrm{M}^{1}, \eta(0)=0\right\} .
$$

here and above $\eta_{s}$ is the distribution derivative of $\eta$ with respect to the internal variable s.

We note that the time-segment integration of the convolution that occurs in the equation $\theta$ results in

import the vector

$$
\left\{\begin{array}{c}
\theta_{t}-\Delta u_{t}-\int_{0}^{\infty} \mu(s) \Delta \eta^{t}(s) d s=\sum_{j=1}^{m} \phi_{j} d \omega_{j} \\
u_{t t}-\Delta u_{t}+\Delta(\Delta u+\theta)+f(u)=0
\end{array}\right.
$$

define the initial value by

$$
b(t)=\left(u(t), v(t), \theta(t), \eta^{t}\right)^{T},
$$

we can transform the studied equation into

$$
b_{0}=\left(u_{0}, v_{0}, \theta_{0}, \eta_{0}\right)^{T} \in V^{0},
$$

$$
\begin{aligned}
& b_{t}=L b+G(b, t, \omega), \\
& b(0)=b_{0}=\left(u_{0}, u_{1}, \theta_{0}, \eta_{0}\right)
\end{aligned}
$$

among them, $G(b, t, \omega)=\left(0,-f(u), \sum_{j=1}^{m} \phi_{j} d \omega_{j}, 0\right)^{T}$, let $u_{t}=v$

$$
L b=L\left(\begin{array}{c}
u \\
v \\
\theta \\
\eta
\end{array}\right)=\left(\begin{array}{c}
v \\
-A v-A(A u-\theta) \\
-A v-\int_{0}^{s} \mu(s) A \eta^{t}(s) d s \\
\theta+T \eta
\end{array}\right)
$$




$$
D(L)=\left\{b \in V^{0} \mid \begin{array}{c}
v, A u-\theta \in v^{2} \\
\theta \in \mathrm{M}^{1} \\
\int_{0}^{s} \mu(s) A \eta^{t}(s) d s \in v^{0} \\
\eta \in D(T)
\end{array}\right\} .
$$

\section{The existence and uniqueness of the solution}

Next, explain the existence, uniqueness, and continuous dependence of the solution on the initial value of the problem (4.2), and show that the solution generates a continuous random dynamic system on probability space $\left(\Omega, F, P,\left(\vartheta_{\mathrm{t}}\right)_{t \in R}\right)$. For this, we consider the one-dimensional Ornstein-Uhlenbeck equation

$$
d z_{j}+z_{j} d t=d \omega_{j}(t), j=1,2, \ldots, m
$$

its solution is the Ornstein-Uhlenbeck process

$$
z_{j}\left(\vartheta_{t} \omega_{j}\right)=-\int_{-\infty}^{0} e^{s}\left(\vartheta_{t} \omega_{j}\right)(s) d s, t \in R
$$

It is known from the literature [2] that the random variable $\left|z_{j}\left(\omega_{j}\right)\right|$ is harmonic, and there is a $\vartheta_{t}$-measure invariant set of all measures, so that for all $\omega \in \Omega$ and $\mathrm{j}=1,2, \ldots, \mathrm{m}, t \mapsto z_{j}\left(\vartheta_{t} \omega_{j}\right)$ are continuous with respect to time $\mathrm{t}$. Therefore, it is known from the proposition 4.3.3 in [2] that there is a harmonic function $r(\omega)>0$ such that

$$
\sum_{j=1}^{m}\left(\left|z_{j}\left(\omega_{j}\right)\right|+\left|z_{j}\left(\omega_{j}\right)\right|^{2}+\left|z_{j}\left(\omega_{j}\right)\right|^{4}+\left|z_{j}\left(\omega_{j}\right)\right|^{6}\right) \leq r(\omega)
$$

among them, $r(\omega)$ satisfies P-a.e. $\omega \in \Omega$

$$
r\left(\vartheta_{t} \omega\right) \leq e^{\frac{1}{2}|t|} r(\omega), t \in R
$$

known from (4.4) and (4.5), for P-a.e. $\omega \in \Omega$

$$
\sum_{j=1}^{m}\left(\left|z_{j}\left(\vartheta_{t} \omega_{j}\right)\right|+\left|z_{j}\left(\vartheta_{t} \omega_{j}\right)\right|^{2}+\left|z_{j}\left(\vartheta_{t} \omega_{j}\right)\right|^{4}+\left|z_{j}\left(\vartheta_{t} \omega_{j}\right)\right|^{6}\right) \leq e^{\frac{1}{2}|t|} r(\omega), t \in R
$$

let

$$
z\left(\vartheta_{t} \omega\right)=z\left(x, \vartheta_{t} \omega\right)=\sum_{j=1}^{m} \phi_{j} z_{j}\left(\vartheta_{t} \omega_{j}\right)
$$

This is a solution to the following equationon

$$
d z+z d t=\sum_{j=1}^{m} \phi_{j} d \omega_{j}
$$

Theorem 4.1 ([10]) Variables $\rho, \rho^{(l)}: \Omega \mapsto R^{+}, l=\frac{1}{2}, 1$, for all $t \in R, \omega \in \Omega$

$$
\begin{aligned}
& \left\|z\left(\vartheta_{t} \omega\right)\right\| \leq e^{\sigma|t|} r(\omega), e^{-\sigma|t|} r(\omega) \leq r\left(\vartheta_{t} \omega\right) \leq e^{\sigma|t|} r(\omega), \\
& \left\|A^{(l)} z\left(\vartheta_{t} \omega\right)\right\| \leq e^{\sigma|t|} r^{(l)}(\omega), e^{-\sigma|t|} r^{(l)}(\omega) \leq r^{(l)}\left(\vartheta_{t} \omega\right) \leq e^{\sigma|t|} r^{(l)}(\omega),
\end{aligned}
$$

among them, $r^{(l)}(\omega)=\sum_{j=1}^{m} r_{j}\left(\omega_{j}\right)\left\|A^{(l)} h_{j}\right\|$. let

Simplify (4.2) to the first-order evolution equation.

$$
\omega=u_{t}+\varepsilon u-z\left(\vartheta_{t} \omega\right), \varphi=\left(u, \omega, \theta, \eta^{t}\right)^{T},
$$

Choose $\varepsilon$ such that $0<\varepsilon<\min \left\{\rho_{1}, \rho_{2}, \rho_{3}\right\}$,

The problem (4.2) is equivalent to the following deterministic dynamic system with random parameters:

$$
\stackrel{\square}{\varphi} H(\varphi)=F\left(\varphi, \vartheta_{t} \omega, t\right), \varphi_{0}(\omega)=\left(u_{0}, u_{1}+\varepsilon u_{0}-z(\omega), \theta_{0}, \eta_{0}\right)^{T}, t \geq 0,
$$


among them,

$$
\begin{gathered}
-H(\varphi)=\left(\begin{array}{c}
\omega-\varepsilon u \\
-A(\omega-\varepsilon u)-A(A u-\theta) \\
-A(\omega-\varepsilon u)-\int_{0}^{\infty} \mu(s) A \eta^{t}(s) d s \\
\theta+T \eta
\end{array}\right)=T_{\varepsilon} L T_{\varepsilon}^{-1} \\
F\left(\varphi, \vartheta_{t} \omega\right)=\left(\begin{array}{c}
z\left(\vartheta_{t} \omega\right) \\
-f(u)-A z\left(\vartheta_{t} \omega\right) \\
-A z\left(\vartheta_{t} \omega\right)+z\left(\vartheta_{t} \omega\right) \\
0
\end{array}\right)
\end{gathered}
$$

It is known from the literature [3] that the operator $\mathrm{L}$ in (4.3) is the infinitesimal generator of the compressed $C_{0}$ semigroup $\left\{e^{L t}\right\}$ in space $V^{0}$, because $-H=T_{\varepsilon} L T_{\varepsilon}^{-1}$ and $\mathrm{L}$ are isomorphic operators in $V^{0}$, So operator $-H$ also generates a compressed $C_{0}$ semigroup $\left\{e^{-H t}\right\}$ in $V^{0}$.

From the conditions satisfied by the nonlinear function $f(u)$ and the embedding relationship $H_{0}^{2}(U) \rightarrow L^{4}(U)$, it is known that function $F\left(\varphi, \vartheta_{t} \omega, t\right)$ is locally Lipschitz continuous with respect to $\varphi$ and $\omega \in \Omega$. Moreover, the time $\mathrm{t}$ is bounded on a finite interval, so $F\left(\varphi, \vartheta_{t} \omega, t\right)$ is continuous with respect to $(\varphi, t)$ and $\mathrm{F}$ is measurable with respect to $\omega$, and the uniqueness of the solution of the differential equation is considered by the classic operator semigroup theory (cf.[3]), We have the following theorem.

Theorem 4.2 If conditions (1)-(4) hold, then for any of $\omega \in \Omega$ and $\varphi_{0} \in V^{0}$, there is $\mathrm{T}>0$, so that when the initial value meets $\varphi\left(0, \omega, \varphi_{0}\right)=\varphi_{0}$, the problem (4.8) has a unique mild solution $\varphi\left(t, \omega, \varphi_{0}\right) \in C\left([0, T) ; V^{0}\right)$ and $\varphi\left(t, \omega, \varphi_{0}\right)$ satisfies

$$
\varphi\left(t, \omega, \varphi_{0}\right)=e^{-H t} \varphi_{0}(\omega)+\int_{0}^{t} e^{-H(t-s)} F\left(s, \vartheta_{s} \omega, \varphi\left(s, \omega, \varphi_{0}\right)\right) d s,
$$

further, $\varphi\left(t, \omega, \varphi_{0}\right)$ is continuous with respect to $t$ and $\varphi_{0}$ and measurable with respect to $\omega$.

Knowing from Theorem 4.2 and the following Theorem 4.3, for any $t \in[0, \infty)$, the solution $\varphi\left(\cdot, \omega, \varphi_{0}\right)$ of equation (4.8) exists globally, therefore, $\varphi\left(\cdot, \omega, \varphi_{0}\right) \in C\left([0,+\infty) ; V^{0}\right)$ defines a continuous random dynamic system from $\mathrm{R}$ to $\left(\Omega, F, P,\left(\vartheta_{\mathrm{t}}\right)_{t \in R}\right)$ :

$$
\begin{aligned}
\Phi(t, \omega): V^{0} & \rightarrow V^{0} \\
\varphi_{0}(\omega) & \mapsto \varphi(t, \omega) \varphi_{0} .
\end{aligned}
$$

In order to prove that the solution of stochastic partial differential equation (1.1) and the solution of partial differential equation (4.8) are conjugated, homeomorphic mapping is introduced

its inverse mapping is

$$
R\left(\vartheta_{t} \omega\right) y=\left(y_{1}, y_{2}+\varepsilon y_{1}-z\left(\vartheta_{t} \omega\right), y_{3}, y_{4}\right)^{T}, y=\left(y_{1}, y_{2}, y_{3}, y_{4}\right)^{T} \in V^{0},
$$

then

$$
R^{-1}\left(\vartheta_{t} \omega\right) y=\left(y_{1}, y_{2}-\varepsilon y_{1}-z\left(\vartheta_{t} \omega\right), y_{3}, y_{4}\right)^{T}
$$

This also determines a continuous random dynamic system.

$$
\Phi(t, \omega)=R\left(\vartheta_{t} \omega\right) S(t, \omega) R^{-1}\left(\vartheta_{t} \omega\right)
$$

On the other hand, if

and

$$
a_{1}=u(t), a_{2}=u_{t}(t), a_{3}=\theta(t), a_{4}=\eta^{t},
$$

$$
a=\left(\begin{array}{l}
a_{1} \\
a_{2} \\
a_{3} \\
a_{4}
\end{array}\right), G(a, \omega)=\left(\begin{array}{c}
0 \\
-f(u) \\
\sum_{j=1}^{m} \phi_{j} d \omega_{j} \\
0
\end{array}\right),
$$


then the partial differential equation (1.1) can be written as

$$
a+H(a)=G(a, \omega), G(a, \omega)=\left(u_{0}, u_{1}, \theta_{0}, \eta_{0}\right)^{T},
$$

let $T y=\left(y_{1}, y_{2}, y_{3}, y_{4}\right)^{T}, y=\left(y_{1}, y_{2}, y_{3}, y_{4}\right)^{T} \in V^{0}$, its inverse map is

$$
T^{-1} y=\left(y_{1}, y_{2}, y_{3}, y_{4}\right)^{T} \text {, }
$$

so

$$
\bar{S}(t, \omega)=T S(t, \omega) T^{-1}
$$

This also determines a continuous random dynamic system $(\vartheta, \bar{S}(t, \omega))$. Therefore, $\bar{S}(t, \omega)$ and $\Phi(t, \omega)$ are equivalent.

Next, the existence of the random attractor of the stochastic dynamic system $\Phi(t, \omega)$ will be studied according to Theorem 2.1.

Theorem 4.3 Assuming that conditions (1)-(4) hold, there is a closed sphere (centered on $0, r_{0}(\omega)$ is radius), so that for any non-random set $B=\{B(\omega)\}_{\omega \in \Omega} \in D$, there is a $T_{B\left(r_{0}(\omega)\right)}>0$, so that for any $\varphi_{0}\left(\vartheta_{-t} \omega\right) \in B\left(\vartheta_{-t} \omega\right)$, the solution $\varphi\left(t, \vartheta_{-t} \omega, \varphi_{0}\right)=\left(u(t), \omega(t), \theta(t), \eta^{t}\right)^{T}$ of equation (4.8) satisfies P-a.e. $\omega \in \Omega$, has

$$
\left\|\varphi\left(t, \vartheta_{-t} \omega, \varphi_{0}\left(\vartheta_{-t} \omega\right)\right)\right\|_{V^{0}}^{2} \leq r_{0}^{2}(\omega) \text {. }
$$

Proof: Use $\varphi(t)=\left(u(t), \omega(t), \theta(t), \eta^{t}\right)^{T}$ and equation (4.8) to make the inner product in $V^{0}$,

among them,

$$
\begin{aligned}
& \frac{d}{d t}\left(\frac{1}{2}\|\varphi\|_{V^{0}}^{2}\right)+\varepsilon\|u\|_{2}^{2}+\|\nabla \omega\|^{2}-\varepsilon(A u, \omega)-\varepsilon(A u, \theta)-\frac{1}{2} \int_{0}^{\infty} \mu^{\prime}(s)\left\|A^{1 / 2} \eta^{\mathrm{t}}(s)\right\|^{2} d s \\
& =\left(\left(z\left(\vartheta_{t} \omega\right), u\right)\right)+\left(-f(u)-A z\left(\vartheta_{t} \omega\right), \omega\right)+\left(-A z\left(\vartheta_{t} \omega\right)+z\left(\vartheta_{t} \omega\right), \theta\right)+0
\end{aligned}
$$

$$
\begin{gathered}
-\frac{1}{2} \int_{0}^{\infty} \mu^{\prime}(s)\left\|A^{1 / 2} \eta^{t}(s)\right\|^{2} d s \geq \frac{\delta}{2}\left\|\eta^{t}\right\|_{\mathrm{M}^{1}}^{2}, \\
\|\nabla \omega\|^{2} \geq D_{1}\|\omega\|^{2}, 2<D_{1}=\left\|A^{1 / 2}\right\|^{2}<\frac{17-\sqrt{65}}{2} .
\end{gathered}
$$

We multiply the second expression in (1.1) by $\varepsilon^{2} u$ and add (4.9), then

$$
\begin{aligned}
& \frac{d}{d t}\left(\frac{1}{2}\|\varphi\|_{V^{0}}^{2}+\frac{\varepsilon^{2}}{2}\|\nabla u\|^{2}+\varepsilon^{2} \int_{U} u u_{t} d x\right)-\varepsilon^{2}\left\|u_{t}\right\|^{2}+\left(\varepsilon+\varepsilon^{2}\right)\|u\|_{2}^{2}+\|\nabla \omega\|^{2} \\
& -\varepsilon(A u, \omega)-\varepsilon(A u, \theta)-\frac{1}{2} \int_{0}^{\infty} \mu^{\prime}(s)\left\|A^{1 / 2} \eta^{\mathrm{t}}(s)\right\|^{2} d s+\varepsilon^{2} \int_{U} u f(u) d x-\varepsilon^{2} \int_{U} \theta A u d x \\
& =\left(\left(z\left(\vartheta_{t} \omega\right), u\right)\right)+\left(-f(u)-A z\left(\vartheta_{t} \omega\right), \omega\right)+\left(-A z\left(\vartheta_{t} \omega\right)+z\left(\vartheta_{t} \omega\right), \theta\right)
\end{aligned}
$$

substitute $\omega=u_{t}+\varepsilon u-z\left(\vartheta_{t} \omega\right)$ into the right side of equation (4.10), the inner product term on the left uses Lemma 2.2, taking the appropriate $\alpha$ has

$$
\begin{aligned}
& \frac{d}{d t}\left(\frac{1}{2}\|\varphi\|_{V^{0}}^{2}+\frac{\varepsilon^{2}}{2}\|\nabla u\|^{2}+\varepsilon^{2} \int_{U} u u_{t} d x-\varepsilon^{2}\|u\|^{2}+\int_{U} F(u) d x\right)+\varepsilon^{2}\|u\|_{2}^{2}+\left(D_{1}-\frac{\varepsilon}{2}\right)\|\omega\|^{2} \\
& -\frac{\varepsilon}{2}\|\theta\|^{2}-\frac{1}{2} \int_{0}^{\infty} \mu^{\prime}(s)\left\|A^{1 / 2} \eta^{t}(s)\right\|^{2} d s-\varepsilon^{2} \int_{U} \theta A u d x+\varepsilon^{2} \int_{U} u f(u) d x \\
& \leq\left(\left(z\left(\vartheta_{t} \omega\right), u\right)\right)-\varepsilon(f(u), u)+\left(f(u), z\left(\vartheta_{t} \omega\right)\right)-\left(A z\left(\vartheta_{t} \omega\right), \omega\right)+\left(-A z\left(\vartheta_{t} \omega\right)+z\left(\vartheta_{t} \omega\right), \theta\right)
\end{aligned}
$$

To estimate the right side of (4.11), from Lemma 2.2(1), take $\alpha=\frac{1}{4}$ and have the following inequality:

$$
\begin{aligned}
\left(\left(z\left(\vartheta_{t} \omega\right), u\right)\right) \leq & \frac{1}{8}\|u\|_{2}^{2}+2\left\|z\left(\vartheta_{t} \omega\right)\right\|_{2}^{2} \\
\left(f(u), z\left(\vartheta_{t} \omega\right)\right) & \leq C_{0} \int_{U}\left(1+|u|^{2}\right)\left|z\left(\vartheta_{t} \omega\right)\right| d x \\
\leq & C_{0} \int_{U}\left|z\left(\vartheta_{t} \omega\right)\right| d x+C_{0}\left(\int_{U}|u|^{3} d x\right)^{\frac{2}{3}}\left\|z\left(\vartheta_{t} \omega\right)\right\|_{L^{3}}
\end{aligned}
$$




$$
\begin{gathered}
\leq C_{0} \int_{U}\left|z\left(\vartheta_{t} \omega\right)\right| d x+C_{0} C_{1}^{-\frac{2}{3}}\left[\frac{2}{3}\left(\varepsilon_{1}\left(\int_{U}\left(F(u)+C_{1}\right) d x\right)^{\frac{2}{3}}\right)^{\frac{3}{2}}+\frac{1}{3 \varepsilon_{1}^{3}}\left\|z\left(\vartheta_{t} \omega\right)\right\|_{L^{3}}^{3}\right. \\
\leq C_{0}\left|z\left(\vartheta_{t} \omega\right)\right|+\kappa \int_{U} F(u) d x+\kappa C_{1}|U|+\tau\left\|z\left(\vartheta_{t} \omega\right)\right\|_{L^{3}}^{3},
\end{gathered}
$$

among them, $\kappa=\frac{2}{3} C_{0} C_{1}^{-\frac{2}{3}} \varepsilon_{1}^{\frac{3}{2}}, \tau=\frac{1}{3 \varepsilon_{1}^{3}}$.

Knowing from the condition (3.5), if $N_{1}>0$ exists, then

$$
(f(u), u) \geq C_{2} \int_{U} F(u) d x-\frac{\lambda_{1}}{8}\|u\|_{2}^{2}-N_{1} .
$$

Use (3.6) to know that there is $0<\delta_{1}<\frac{1}{2}, I=I\left(\delta_{1}\right)>0$ and get $f(s) s \geq-\frac{1-\delta_{1}}{C_{U}} s^{2}, \forall|s| \geq N$. Then

$$
\int_{U} f(u) u d x \geq-\left(1-\delta_{1}\right)\|u\|_{2}^{2}+C(|U|, f)
$$

among them, $C(|U|, f)=|U| \min _{|s| \leq N} F(s)$.

Synthesizing the above inequalities and using Lemma 2.2, take the appropriate $\alpha$ to get

$$
\begin{aligned}
& \frac{d}{d t}\left(\frac{1}{2}\|\varphi\|_{V^{0}}^{2}+\frac{\varepsilon^{2}}{2}\|\nabla u\|^{2}+\varepsilon^{2} \int_{U} u u_{t} d x-\varepsilon^{2}\|u\|^{2}+\int_{U} F(u) d x\right)+\left(\varepsilon^{2} \delta_{1}-\frac{\lambda_{1} \varepsilon+1}{8}-\frac{\varepsilon^{2}}{2}\right)\|u\|_{2}^{2} \\
& +\left(D_{1}-\frac{\varepsilon}{2}-\frac{1}{8}\right)\|\omega\|^{2}-\left(\frac{\varepsilon}{2}+\frac{1}{4}+\frac{\varepsilon^{2}}{2}\right)\|\theta\|^{2}-\frac{1}{2} \int_{0}^{\infty} \mu^{\prime}(s)\left\|A^{1 / 2} \eta^{\mathrm{t}}(s)\right\|^{2} d s+\left(\varepsilon C_{2}-\kappa\right) \int_{U} F(u) d x \\
& \leq 6\left\|A z\left(\vartheta_{t} \omega\right)\right\|^{2}+C_{0}\left|z\left(\vartheta_{t} \omega\right)\right|+\kappa C_{1}|U|+\tau\left\|z\left(\vartheta_{t} \omega\right)\right\|_{L^{3}}^{3}+2\left\|z\left(\vartheta_{t} \omega\right)\right\|^{2}-\varepsilon^{2} C(|U|, f)+\varepsilon N_{1}
\end{aligned}
$$

Let $J(t)=-\int_{0}^{\infty} \mu(s)\left\langle\theta(t), \eta^{t}(s)\right\rangle d s$, then $|J(t)| \leq\|\theta(t)\| \int_{0}^{\infty} \mu(s)\left\langle\eta^{t}(s), \eta^{t}(s)\right\rangle^{\frac{1}{2}} d s \leq C\|\varphi\|_{V^{0}}^{2}$.

$\frac{d}{d t} J(t)=-\int_{0}^{\infty} \mu(s)\left\langle\theta_{t}(t), \eta^{t}(s)\right\rangle d s-\int_{0}^{\infty} \mu(s)\left\langle\theta(t), \eta_{t}^{t}(s)\right\rangle d s$

$=-\int_{0}^{\infty} \mu(s)\left\langle-A \omega, \eta^{t}(s)\right\rangle d s-\int_{0}^{\infty} \mu(s)\left\langle\varepsilon A u, \eta^{t}(s)\right\rangle d s+\int_{0}^{\infty} \mu(s)\left\langle A z\left(\vartheta_{t} \omega\right), \eta^{t}(s)\right\rangle d s$

Estimation

$+\int_{0}^{\infty} \mu(s)\left\langle\int_{0}^{\infty} \mu(s) A \eta^{t}(s) d s, \eta^{t}(s)\right\rangle d s-\int_{0}^{\infty} \mu(s)\left\langle z\left(\vartheta_{t} \omega\right), \eta^{t}(s)\right\rangle d s-k_{0}\|\theta\|^{2}+\int_{0}^{\infty} \mu(s)\left\langle\theta(t), \eta_{s}^{t}(s)\right\rangle d s$

of the above formula has the following inequality

$$
\int_{0}^{\infty} \mu(s)\left\langle\int_{0}^{\infty} \mu(s) A \eta^{t}(s) d s, \eta^{t}(s)\right\rangle d s \leq k_{0}\left\|\eta^{t}\right\|_{M^{1}}^{2},
$$

At the same time using Lemma 2.2 (3)(4), take the appropriate $\alpha$ to get

$$
\begin{aligned}
& \frac{d}{d t} J(t)+\frac{3 k_{0}}{4}\|\theta\|^{2} \leq \frac{k_{0} D_{1}}{4}\|\omega\|^{2}+\frac{k_{0} \varepsilon}{4}\|u\|_{2}^{2}+\left(\frac{1+4 \varepsilon}{4 D_{1}}+k_{0}+\frac{5}{4}\right)\left\|\eta^{t}\right\|_{M^{1}}^{2} \\
& -\frac{1}{D_{1}} \int_{0}^{\infty} \mu^{\prime}(s)\left\|A^{1 / 2} \eta^{t}(s)\right\|^{2} d s+k_{0}\left\|\nabla z\left(\vartheta_{t} \omega\right)\right\|^{2}+k_{0}\left\|z\left(\vartheta_{t} \omega\right)\right\|^{2} .
\end{aligned}
$$

Next, add (4.13) and (4.12) to get

$\frac{d}{d t}\left(\frac{1}{2}\|\varphi\|_{V^{0}}^{2}+\frac{\varepsilon^{2}}{2}\|\nabla u\|^{2}+\varepsilon^{2} \int_{U} u u_{t} d x-\varepsilon^{2}\|u\|^{2}+\int_{U} F(u) d x+J(t)\right)+\left(D_{1}-\frac{\varepsilon}{2}-\frac{k_{0} \varepsilon}{4}-\frac{1}{8}\right)\|\omega\|^{2}$

$+\left(\varepsilon^{2} \delta_{1}-\frac{\lambda_{1} \varepsilon+1}{8}-\frac{\varepsilon^{2}}{2}-\frac{k_{0} \varepsilon}{4}\right)\|u\|_{2}^{2}+\left(\frac{3 k_{0}-1}{4}-\frac{\varepsilon+\varepsilon^{2}}{2}\right)\|\theta\|^{2}+\left(\varepsilon C_{2}-\kappa\right) \int_{U} F(u) d x$

$+\left(\frac{\delta}{2}-\frac{\delta+\varepsilon}{D_{1}}-\frac{5}{4}-\frac{1}{4 D_{1}}-k_{0}\right)\left\|\eta^{t}\right\|_{M^{1}}^{2}$

$\leq k_{0}\left\|\nabla z\left(\vartheta_{t} \omega\right)\right\|^{2}+6\left\|A z\left(\vartheta_{t} \omega\right)\right\|^{2}+C_{0}\left|z\left(\vartheta_{t} \omega\right)\right|+\kappa C_{1}|U|+\tau\left\|z\left(\vartheta_{t} \omega\right)\right\|_{L^{3}}^{3}+\left(2+k_{0}\right)\left\|z\left(\vartheta_{t} \omega\right)\right\|^{2}$

$-\varepsilon^{2} C(|U|, f)+\varepsilon N_{1}$ 
among them

$$
-\int_{0}^{\infty} \mu^{\prime}(s)\left\|A^{1 / 2} \eta^{t}(s)\right\|^{2} d s \geq \delta \int_{0}^{\infty} \mu(s)\left\|A^{1 / 2} \eta^{t}(s)\right\|^{2} d s=\delta\left\|\eta^{t}\right\|_{M^{1}}^{2} .
$$

Let

$$
\begin{aligned}
& R_{0}\left(\vartheta_{t} \omega\right)=\left\|\nabla z\left(\vartheta_{t} \omega\right)\right\|^{2}+\left|z\left(\vartheta_{t} \omega\right)\right|+\left\|z\left(\vartheta_{t} \omega\right)\right\|_{L^{3}}^{3}+\left\|A z\left(\vartheta_{t} \omega\right)\right\|^{2}+\left\|z\left(\vartheta_{t} \omega\right)\right\|^{2}, \\
& G(t)=\frac{1}{2}\|\varphi\|_{V^{0}}^{2}+\frac{\varepsilon^{2}}{2}\|\nabla u\|^{2}+\varepsilon^{2} \int_{U} u u_{t} d x-\varepsilon^{2}\|u\|^{2}+\int_{U} F(u) d x+J(t),
\end{aligned}
$$

There is

$$
\frac{d}{d t} G(t)+\beta\|\varphi\|_{V^{0}}^{2} \leq \frac{d}{d t} G(t)+\beta\|\varphi\|_{V^{0}}^{2}+\left(\varepsilon C_{2}-\kappa\right) \int_{U} F(u) d x \leq \chi
$$

among them,

$Q=\max \left\{6, C_{0}, 2+k_{0}, \tau\right\}$,

$\chi=Q R_{0}\left(\vartheta_{t} \omega\right)+\kappa C_{1}|U|-\varepsilon^{2} C(|U|, f)+\varepsilon N_{1}$,

(within the

$\beta=\min \left\{\varepsilon^{2} \delta_{1}-\frac{\lambda_{1} \varepsilon+1}{8}-\frac{\varepsilon^{2}}{2}-\frac{k_{0} \varepsilon}{4}, D_{1}-\frac{\varepsilon}{2}-\frac{k_{0} \varepsilon}{4}-\frac{1}{8}, \frac{3 k_{0}-1}{4}-\frac{\varepsilon+\varepsilon^{2}}{2}, \frac{\delta}{2}-\frac{\delta+\varepsilon}{D_{1}}-\frac{5}{4}-\frac{1}{4 D_{1}}-k_{0}\right\}$

limited range of each parameter, each item in $\beta$ is greater than zero).

$$
\text { Let } \rho_{1}=\min \left\{\frac{\lambda_{1}+2 k_{0}-\sqrt{\frac{\lambda_{1}^{2}}{64}+\frac{\lambda_{1} k_{0}+k_{0}^{2}}{16}+\frac{\delta_{1}}{2}-\frac{1}{4}}}{16 \delta_{1}-8}, \frac{8 D_{1}-1}{4\left(k_{0}+1\right)},-\frac{1}{2}+\sqrt{\frac{1}{8}+\frac{3 k_{0}}{2}}, \frac{\delta}{2} D_{1}-\delta-\frac{5 D_{1}+1}{4}-k_{0} D_{1}\right\} \text {. }
$$

(within the limited range of each parameter, each item in $\rho_{1}$ is greater than zero).

By Theorem 4.1

$$
\int_{-t}^{0} R_{0}\left(\vartheta_{s} \omega\right) e^{\beta s} d s \leq \int_{-t}^{0} \tilde{R}_{0}(s, \omega) e^{\beta s} d s \leq \int_{-\infty}^{0} \tilde{R}_{0}(s, \omega) e^{\beta s} d s<+\infty
$$

among them,

$$
\tilde{R}_{0}(s, \omega)=e^{2 \sigma|t|} r^{2}(\omega)+e^{\frac{1}{2}|t|} r(\omega)+\left(e^{\sigma|t|} r(\omega)\right)^{3}+\left(e^{\sigma|t|} r^{(1)}(\omega)\right)^{2}+\left(e^{\sigma|t|} r^{(1 / 2)}(\omega)\right)^{2} .
$$

By Lemma 2.1 there is $G\left(\varphi\left(t, \omega, \varphi_{0}(\omega)\right) \leq \sup _{\xi \in V^{0}}\left\{E(\xi): \beta\|\xi\|_{V^{0}}^{2} \leq \chi+\varepsilon_{1}\right\}\right.$.

Use $\vartheta_{-t} \omega$ instead of $\omega$ in the above formula to get

$$
G\left(\varphi\left(t, \vartheta_{-t} \omega, \varphi_{0}\left(\vartheta_{-t} \omega\right)\right) \leq \sup _{\xi \in V^{0}}\left\{E(\xi): \beta\|\xi\|_{V^{0}}^{2} \leq \chi+\varepsilon_{1}\right\}\right.
$$

Then

$$
\| \varphi\left(t, \vartheta_{-t} \omega, \varphi_{0}\left(\vartheta_{-t} \omega\right) \|_{V^{0}}^{2} \leq \frac{\chi+\varepsilon_{1}}{\beta}=r_{0}^{2}(\omega)\right.
$$

To sum up, the theorem is proved.

In order to obtain the regularity results of the stochastic dynamic system $\Phi(t, \omega)$, using a method similar to [1], the solution $\varphi=\left(u, \omega, \theta, \eta^{t}\right)^{T}$ of the system is decomposed into $\varphi=\varphi_{L}+\varphi_{N}$, where $\varphi_{L}=\left(u_{L}, \omega_{L}, \theta_{L}, \eta_{L}^{t}\right)^{T}$ and $\varphi_{N}=\left(u_{N}, \omega_{N}, \theta_{N}, \eta_{N}^{t}\right)^{T}$ are the solutions of the following equations

$$
\stackrel{\sqcap}{\varphi_{L}}+H\left(\varphi_{L}\right)=0, \varphi_{L}(0, \omega)=\left(u_{0}, u_{1}+\varepsilon u_{0}, \theta_{0}, \eta_{0}\right)^{T}, t \geq 0
$$

and

among them,

$$
\stackrel{[}{\varphi_{N}}+H\left(\varphi_{N}\right)=F_{2}(\omega), \varphi_{N}(0, \omega)=(0,-z(\omega), 0,0)^{T}, t \geq 0
$$

$$
F_{2}(\omega)=\left(\begin{array}{c}
z\left(\vartheta_{t} \omega\right) \\
-f(u)-A z\left(\vartheta_{t} \omega\right) \\
z\left(\vartheta_{t} \omega\right)-A z\left(\vartheta_{t} \omega\right) \\
0
\end{array}\right) .
$$

For equations (4.15) and (4.16) there are regular results as follows. 
Theorem 4.4 Assuming conditions (1)-(4) hold, let $B \subset V^{0}$ be a non-random bounded set, then for any $\varphi_{L}(0, \omega)=\left(u_{0}, u_{1}+\varepsilon u_{0}, \theta_{0}, \eta_{0}\right)^{T} \in B$, the following holds

$$
\left\|\varphi_{L}\left(t, \vartheta_{-t} \omega, \varphi_{L}\left(0, \vartheta_{-t} \omega\right)\right)\right\|_{V^{0}}^{2}=\left\|u_{L}\right\|_{2}^{2}+\left\|\omega_{L}\right\|^{2}+\left\|\theta_{L}\right\|^{2}+\left\|\eta_{L}^{t}\right\|_{\mathrm{M}^{1}}^{2} \rightarrow 0,(t \rightarrow \infty),
$$

$\varphi_{L}=\left(u_{L}, \omega_{L}, \theta_{L}, \eta_{L}^{t}\right)^{T}$ meet equation (4.15).

Proof: In space $V^{0}$, use $\varphi_{L}=\left(u_{L}, \omega_{L}, \theta_{L}, \eta_{L}^{t}\right)^{T}$ and (4.15) as the inner product, where $\omega_{L}=u_{L t}+\varepsilon u_{L}$, the corresponding initial value is $\left(u_{0}, u_{1}+\varepsilon u_{0}, \theta_{0}, \eta_{0}\right)^{T}$.

$$
\frac{1}{2} \frac{d}{d t}\left\|\varphi_{L}\right\|_{V^{0}}^{2}+\left(H\left(\varphi_{L}\right), \varphi_{L}\right)_{V^{0}}=0
$$

among them, $\left(H\left(\varphi_{L}\right), \varphi_{L}\right)_{V^{0}} \geq \frac{\varepsilon}{2}\left\|u_{L}\right\|^{2}+\left(D_{1}-\varepsilon\right)\left\|\omega_{L}\right\|^{2}-\varepsilon\left\|\theta_{L}\right\|^{2}+\frac{\delta}{2}\left\|\eta_{L}^{t}\right\|^{2}$.

Define the energy functional:

$$
\begin{gathered}
\mathrm{K}(t)=\int_{U} \omega_{L}(t) u_{L}(t) d x \\
\mathrm{P}(t)=\int_{U} \theta_{L}(t) u_{L}(t) d x \\
\Psi(t)=-\int_{U} \theta_{L}(t) \int_{0}^{\infty} \mu(s) \eta_{L}^{t}(s) d s d x .
\end{gathered}
$$

Then derivate $\mathrm{K}(t), \mathrm{P}(t))$ and $\Psi(t)$ with respect to time t, and use the Herder's inequality to get

$$
\begin{aligned}
\frac{d}{d t} \mathrm{~K}(t) & =\int_{U} \omega_{L t}(t) u_{L}(t) d x+\int_{U} \omega_{L}(t) u_{L t}(t) d x \\
& =\int_{U}\left(-A\left(\omega_{L}-\varepsilon u_{L}\right)-A\left(A u_{L}-\theta_{L}\right)\right) u_{L} d x+\left\|\omega_{L}\right\|^{2}-\varepsilon \int_{U} \omega_{L} u_{L} d x \\
& \leq\left(2-\frac{\varepsilon}{4}\right)\left\|\omega_{L}\right\|^{2}+\left(\frac{\varepsilon\left(D_{1}-1\right)}{D_{1}^{2}}-\frac{1}{2}\right)\left\|u_{L}\right\|_{2}^{2}+\frac{1}{2}\left\|\theta_{L}\right\|^{2}+C . \\
\frac{d}{d t} \mathrm{P}(t) & =\int_{U} \theta_{L t}(t) u_{L}(t) d x+\int_{U} \theta_{L}(t) u_{L t}(t) d x \\
& =\int_{U}\left(-A\left(\omega_{L}-\varepsilon u_{L}\right)-\int_{0}^{\infty} \mu(s) A \eta_{L}^{t}(s) d s\right) u_{L} d x+\int_{U} \theta_{L}\left(\omega_{L}-\varepsilon u_{L}\right) d x \\
& \leq-\int_{U} A \omega_{L} u_{L} d x+\varepsilon D_{1}\left\|u_{L}\right\|_{2}^{2}-\int_{0}^{\infty} \mu(s)\left(\int_{U} A u_{L} \eta_{L}^{t}(s) d x\right) d s+\int_{U} \theta_{L} \omega_{L} d x-\varepsilon \int_{U} \theta_{L} u_{L} d x \\
& \leq\left\|\omega_{L}\right\|^{2}+\left(\frac{1+k_{0}}{2}+\frac{\varepsilon\left(D_{1}+1\right)}{D_{1}^{2}}\right)\left\|u_{L}\right\|_{2}^{2}+\frac{1}{2 D_{1}}\left\|\eta_{L}^{t}\right\|_{\mathrm{M}^{1}}^{2}+\frac{2+\varepsilon}{4}\left\|\theta_{L}\right\|^{2}+C .
\end{aligned}
$$

Then take the derivative of $\Psi(t)$,

$$
\begin{aligned}
\frac{d}{d t} \Psi(t) & =-\frac{d}{d t} \int_{U} \theta_{L}(t) \int_{0}^{\infty} \mu(s) \eta_{L}^{t}(s) d s d x \\
= & -\int_{U} \theta_{L t}(t) \int_{0}^{\infty} \mu(s) \eta_{L}^{t}(s) d s d x-\int_{U} \theta_{L}(t) \int_{0}^{\infty} \mu(s) \eta_{L t}^{t}(s) d s d x \\
= & \int_{U} A \omega_{L} \int_{0}^{\infty} \mu(s) \eta_{L}^{t}(s) d s d x-\varepsilon \int_{U} A u_{L} \int_{0}^{\infty} \mu(s) \eta_{L}^{t}(s) d s d x \\
& \left.+\int_{U} \int_{0}^{\infty} \mu(s) A \eta_{L}^{t}(s) d s\right) \int_{0}^{\infty} \mu(s) \eta_{L}^{t}(s) d s d x-\int_{U} \theta_{L}(t) \int_{0}^{\infty} \mu(s) \eta_{L t}(s) d s d x
\end{aligned}
$$

among them,

$$
\left.\mid \int_{U} \int_{0}^{\infty} \mu(s) A \eta_{L}^{t}(s) d s\right) \int_{0}^{\infty} \mu(s) \eta_{L}^{t}(s) d s d x \mid \leq k_{0}\left\|\eta_{L}^{t}\right\|_{\mathrm{M}^{1}}^{2}
$$


$-\int_{U} \theta_{L}(t) \int_{0}^{\infty} \mu(s) \eta_{L t}^{t}(s) d s d x$

$\leq-k_{0}\left\|\theta_{L}\right\|^{2}+\int_{U}\left|\theta_{L}\right|\left(\int_{0}^{s_{0}} \frac{\left|\mu^{\prime}(s)\right|}{\mu^{1 / 2}\left(s_{0}\right)} \mu^{1 / 2}(s)\left|\eta_{L}^{t}(s)\right| d s+M \int_{0}^{\infty} \mu(s)\left|\eta_{L}^{t}(s)\right| d s\right) d x$

$\leq-\left(k_{0}-\frac{1}{4}\right)\left\|\theta_{L}\right\|^{2}+C\left\|\eta_{L}^{t}\right\|_{\mathrm{M}^{1}}^{2}$,

among them, $C=\frac{\sqrt{2}}{\lambda_{1}} \max \left\{\frac{1}{\mu^{1 / 2}\left(s_{0}\right)}\left(\int_{0}^{s_{0}}\left|\mu^{\prime}(s)\right|^{2} d s\right)^{1 / 2}, M \mu_{0}^{1 / 2}\right\}$.

Then,

$$
\frac{d}{d t} \Psi(t) \leq \frac{\varepsilon}{4}\left\|u_{L}\right\|_{2}^{2}+\frac{1}{4}\left\|\omega_{L}\right\|^{2}-\left(k_{0}-\frac{1}{4}\right)\left\|\theta_{L}\right\|^{2}+\left(C+D_{1}+\frac{\varepsilon}{D_{1}}+k_{0}\right)\left\|\eta_{L}^{t}\right\|_{\mathrm{M}^{1}}^{2} .
$$

Let, $L(t)=M Y(t)+\gamma(\mathrm{K}(t)+\mathrm{P}(t))+\Psi(t)$,

among them

$\gamma<l$,

$l=\min \left\{\frac{D_{1}\left(4 k_{0}-1\right)-4 k_{0} \varepsilon}{-2 \varepsilon^{2}+\varepsilon\left(8+D_{1}\right)+4 D_{1}}, \frac{D_{1}\left(4 k_{0}-1-2 \varepsilon\right)}{16 \varepsilon+8 k_{0} D_{1}+4 D_{1}+\varepsilon D_{1}}, \frac{\varepsilon D_{1}\left(\delta-4 k_{0}\right)-4 \varepsilon\left(\varepsilon+D_{1} C+D_{1}^{2}\right)}{2 \varepsilon+2 k_{0} \delta D_{1}-8 \delta \varepsilon}\right\}$,

$\varsigma<M<\frac{4\left(k_{0}-\gamma\right)-1-\varepsilon \gamma}{4 \varepsilon}$,

$\varsigma=\max \left\{\frac{12 \gamma+1-\varepsilon \gamma}{4\left(D_{1}-\varepsilon\right)}, \frac{2}{\delta}\left(C+D_{1}+k_{0}+\frac{\varepsilon}{D_{1}}+\frac{\gamma}{2 D_{1}}\right), \frac{1}{2}+\frac{4 \gamma}{D_{1}}+\frac{\gamma k_{0}}{\varepsilon}\right\}$.

(within the limited range of each parameter, each item in $\varsigma$ is less than $\frac{4\left(k_{0}-\gamma\right)-1-\varepsilon \gamma}{4 \varepsilon}$ )

The inequalities obtained by integrating the results of the above three functions are

$$
\begin{aligned}
& \frac{d}{d t} L(t)=M \frac{d}{d t} Y(t)+\gamma \frac{d}{d t}(\mathrm{~K}(t)+\mathrm{P}(t))+\frac{d}{d t} \Psi(t) \\
& \leq\left(\varepsilon M-M D_{1}+3 \gamma+\frac{1-\varepsilon \gamma}{4}\right)\left\|\omega_{L}\right\|^{2}+\left(\frac{\varepsilon}{D_{1}}-\frac{\delta M}{2}+C+D_{1}+\frac{\gamma}{2 D_{1}}+k_{0}\right)\left\|\eta_{L}^{t}\right\|_{M^{1}}^{2} \\
& \quad+\left(\frac{\varepsilon}{4}+\frac{2 \varepsilon \gamma}{D_{1}}+\frac{\gamma k_{0}-\varepsilon M}{2}\right)\left\|u_{L}\right\|_{2}^{2}+\left(\gamma+\varepsilon M-k_{0}+\frac{\gamma \varepsilon+1}{4}\right)\left\|\theta_{L}\right\|^{2},
\end{aligned}
$$

let,

$\beta_{1}=\min \left\{M D_{1}-\varepsilon M-3 \gamma-\frac{1-\varepsilon \gamma}{4}, \frac{\varepsilon M}{2}-\frac{\varepsilon}{4}-\frac{2 \varepsilon \gamma}{D_{1}}-\frac{\gamma k_{0}}{2}, k_{0}-\gamma-\varepsilon M-\frac{\gamma \varepsilon+1}{4}, \frac{\delta M}{2}-\frac{\varepsilon}{D_{1}}-C-D_{1}-\frac{\gamma}{2 D_{1}}-k_{0}\right\}$,

$\rho_{2}=\min \left\{D_{1}, \frac{72+D_{1}^{2}-15 D_{1}}{4}, \frac{k_{0} \delta D_{1}}{4 \delta-1}\right\}$.

(within

the limited range of each parameter, each item in $\beta_{1}$ and $\rho_{2}$ is greater than zero).

Then

$$
\frac{d}{d t} L(t)+\beta_{1} Y(t) \leq 0,
$$

applying Gronwall's lemma to the above formula

$$
L\left(t, \omega, \varphi_{L}(0, \omega)\right) \leq Y\left(0, \omega, \varphi_{L}(0, \omega) e^{-\beta_{1} t},\right.
$$

use $\vartheta_{-t} \omega$ instead of $\omega$ in the above formula to get

$$
L\left(t, \vartheta_{-t} \omega, \varphi_{L}\left(0, \vartheta_{-t} \omega\right)\right) \leq Y\left(0, \vartheta_{-t} \omega, \varphi_{L}\left(0, \vartheta_{-t} \omega\right) e^{-\beta_{1} t},\right.
$$

So,

$2 Y\left(t, \vartheta_{-t} \omega, \varphi_{L}\left(0, \vartheta_{-t} \omega\right) \leq 2 C L\left(t, \vartheta_{-t} \omega, \varphi_{L}\left(0, \vartheta_{-t} \omega\right)\right)\right)$

$$
\begin{aligned}
& \leq 2 C Y\left(0, \vartheta_{-t} \omega, \varphi_{L}\left(0, \vartheta_{-t} \omega\right)\right) e^{-\beta_{1} t} \\
& \leq 2 C e^{-\beta_{1} t}\left(\left\|u_{0}\right\|_{2}^{2}+\left\|u_{1}+\varepsilon u_{0}\right\|^{2}+\left\|\theta_{0}\right\|^{2}+\left\|\eta_{0}\right\|_{M^{1}}^{2}\right) \rightarrow 0 .(t \rightarrow \infty) .
\end{aligned}
$$


To sum up, the theorem is proved.

Theorem 4.5 Assuming that conditions (1)-(4) hold, there is a random closed sphere $B=\{B(\omega)\}_{\omega \in \Omega} \in D$ centered on 0 and radiused $r_{1}(\omega)>0$, while $\bar{B}(\omega) \in D, \varphi_{0}(\omega) \in \bar{B}(\omega)$ and $T_{\bar{B}\left(r_{1} \omega\right)}>0$ exist, so that for any $\varphi_{N}=\left(u_{N}, \omega_{N}, \theta_{N}, \eta_{N}^{t}\right)^{T}$, the system (4.16) satisfies P-a.e. $\omega \in \Omega$, there is

$$
\left\|A^{r / 2} u_{N}\right\|_{2}^{2}+\left\|A^{r / 2} \omega_{N}\right\|^{2}+\left\|A^{r / 2} \theta_{N}\right\|^{2}+\left\|A^{r / 2} \eta_{N}^{t}\right\|_{\mathrm{M}^{1}}^{2} \leq r_{1}^{2}(\omega) .
$$

Proof: Knowing from Theorem 4.3, Theorem 4.4 and $\varphi_{N}=\varphi-\varphi_{L}$, there is a positive random variable $M_{3}(\omega)>0$, making

$$
\max \left\{\left\|\varphi\left(0, \vartheta_{-t} \omega, \varphi\left(0, \vartheta_{-t} \omega\right)\right)\right\|_{V^{0}},\left\|\varphi_{N}\left(0, \vartheta_{-t} \omega, \varphi_{N}\left(0, \vartheta_{-t} \omega\right)\right)\right\|_{V^{0}}\right\} \leq M_{3}(\omega)
$$

In space $V^{0}$, use $A^{r} \varphi_{N}=\left(A^{r} u_{N}, A^{r} \omega_{N}, A^{r} \theta_{N}, A^{r} \eta_{N}^{t}\right)^{T}$ and (4.16) as the inner product

$$
\begin{aligned}
& \frac{1}{2}\left(\left\|A^{r / 2} u_{N}\right\|_{2}^{2}+\left\|A^{r / 2} \omega_{N}\right\|^{2}+\left\|A^{r / 2} \theta_{N}\right\|^{2}+\left\|A^{r / 2} \eta_{N}^{t}\right\|_{M^{1}}^{2}\right)+\left(H\left(\varphi_{N}\right), A^{r} \varphi_{N}\right)_{V^{0}} \\
& =\left(\left(z\left(\vartheta_{t} \omega\right), A^{r} u_{N}\right)\right)-\left(f(u), A^{r} \omega_{N}\right)-\left(A z\left(\vartheta_{t} \omega\right), A^{r} \omega_{N}\right)-\left(A z\left(\vartheta_{t} \omega\right), A^{r} \theta_{N}\right)+\left(z\left(\vartheta_{t} \omega\right), A^{r} \theta_{N}\right)
\end{aligned}
$$

It can be calculated by the similar theorem 4.4

$$
\left(H\left(\varphi_{N}\right), A^{r} \varphi_{N}\right)_{V^{0}} \geq \frac{\varepsilon}{2}\left\|A^{r / 2} u_{N}\right\|_{2}^{2}+\left(D_{1}-\varepsilon\right)\left\|A^{r / 2} \omega_{N}\right\|^{2}-\varepsilon\left\|A^{r / 2} \theta_{N}\right\|^{2}+\frac{\delta}{2}\left\|A^{r / 2} \eta_{N}^{t}\right\|^{2}
$$

Next estimate each item on the right side of (4.18), using the condition (3.6), $f(u) \geq-\frac{1-\delta_{1}}{C_{U}} u$, we get

$$
\left|-\left(f(u), A^{r} \omega_{N}\right)\right| \leq \frac{\left(1-\delta_{1}\right)^{2} C_{s}}{4 C_{U}^{2}} r_{0}^{2}(\omega)+\left\|A^{r / 2} \omega_{N}\right\|^{2}
$$

where $C_{s}>0$ is the embedding constant.

To sum up, using Lemma 2.2, take the appropriate $\alpha$ to get,

$$
\begin{aligned}
& \frac{d}{d t}\left\|A^{r / 2} \varphi_{N}\right\|_{V^{0}}^{2}+\left(\varepsilon-\frac{1}{2}\right)\left\|A^{r / 2} u_{N}\right\|_{2}^{2}+\left(2 D_{1}-2 \varepsilon-\frac{5}{2}\right)\left\|A^{r / 2} \omega_{N}\right\|^{2}-\left(2 \varepsilon+\frac{1}{2}\right)\left\|A^{r / 2} \theta_{N}\right\|^{2}+\delta\left\|A^{r / 2} \eta_{N}^{t}\right\|^{2} \\
& \leq 2\left\|A^{r / 2} z\left(\vartheta_{t} \omega\right)\right\|_{2}^{2}+\frac{\left(1-\delta_{1}\right)^{2} C_{s}}{2 C_{U}^{2}} r_{0}^{2}(\omega)+6\left\|A^{(r+2) / 2} z\left(\vartheta_{t} \omega\right)\right\|^{2}+4\left\|A^{r / 2} z\left(\vartheta_{t} \omega\right)\right\|^{2}
\end{aligned}
$$

Let $A^{r} J_{N}(t)=-\int_{0}^{\infty} \mu(s) A^{r}\left\langle\theta_{N}, \eta_{N}^{t}(s)\right\rangle d s$, then

$$
\left|A^{r} J_{N}(t)\right| \leq\left\|A^{r / 2} \theta_{N}\right\| \int_{0}^{\infty} \mu(s) A^{r / 2}\left\langle\eta_{N}^{t}(s), \eta_{N}^{t}(s)\right\rangle^{\frac{1}{2}} d s \leq C_{n}\left\|A^{r / 2} \varphi_{N}\right\|_{V^{0}}^{2} .
$$

By Theorem 4.3 there

$$
\begin{aligned}
& \frac{d}{d t} A^{r} J_{N}(t)+\frac{3 k_{0}}{4}\left\|A^{r / 2} \theta_{N}\right\|^{2} \leq \frac{k_{0} D_{1}}{4}\left\|A^{r / 2} \omega_{N}\right\|^{2}+\frac{k_{0} \varepsilon}{4}\left\|A^{r / 2} u_{N}\right\|_{2}^{2}+\left(\frac{1+4 \varepsilon+4 \delta}{4 D_{1}}+k_{0}+\frac{5}{4}\right)\left\|A^{r / 2} \eta_{N}^{t}\right\|_{M^{1}}^{2} \\
& +k_{0}\left\|A^{(1+r) / 2} z\left(\vartheta_{t} \omega\right)\right\|^{2}+k_{0}\left\|A^{r} z\left(\vartheta_{t} \omega\right)\right\|^{2} .
\end{aligned}
$$

Combining (4.19) and (4.20)

$$
\begin{aligned}
& \frac{d}{d t}\left(\left\|A^{r / 2} \varphi_{N}\right\|_{V^{0}}^{2}+A^{r} J_{N}(t)\right)+\left(\varepsilon-\frac{1}{2}-\frac{k_{0} \varepsilon}{4}\right)\left\|A^{r / 2} u_{N}\right\|_{2}^{2}+\left(2 D_{1}-2 \varepsilon-\frac{5}{2}-\frac{k_{0} D_{1}}{4}\right)\left\|A^{r / 2} \omega_{N}\right\|^{2} \\
& +\left(\frac{3 k_{0}}{4}-2 \varepsilon-\frac{1}{2}\right)\left\|A^{r / 2} \theta_{N}\right\|^{2}+\left(\delta-\frac{1+4 \varepsilon+4 \delta}{4 D_{1}}-k_{0}-\frac{5}{4}\right)\left\|A^{r / 2} \eta_{N}^{t}\right\|^{2} \\
& \leq 2\left\|A^{r / 2} z\left(\vartheta_{t} \omega\right)\right\|_{2}^{2}+\frac{\left(1-\delta_{1}\right)^{2} C_{s}}{2 C_{U}^{2}} r_{0}^{2}(\omega)+6\left\|A^{(r+2) / 2} z\left(\vartheta_{t} \omega\right)\right\|^{2}+\left(k_{0}+4\right)\left\|A^{r / 2} z\left(\vartheta_{t} \omega\right)\right\|^{2}+k_{0}\left\|A^{(1+r) / 2} z\left(\vartheta_{t} \omega\right)\right\|^{2} . \\
& \text { Let } X(t)=\left\|A^{r / 2} \varphi_{N}\right\|_{V^{0}}^{2}=\left\|A^{r / 2} u_{N}\right\|_{2}^{2}+\left\|A^{r / 2} \omega_{N}\right\|^{2}+\left\|A^{r / 2} \theta_{N}\right\|^{2}+\left\|A^{r / 2} \eta_{N}^{t}\right\|_{M^{1}}^{2}
\end{aligned}
$$

There is

$$
\left(1-C_{n}\right) \frac{d}{d t} X(t)+\beta_{2} X(t) \leq \frac{d}{d t}\left(\left\|A^{r / 2} \varphi_{N}\right\|_{V^{0}}^{2}+A^{r} J_{N}(t)\right)+\beta_{2} X(t) \leq P R_{1}\left(\vartheta_{t} \omega\right),
$$


among them,

$$
\begin{aligned}
& R_{1}\left(\vartheta_{t} \omega\right)=\left\|A^{(1+r) / 2} z\left(\vartheta_{t} \omega\right)\right\|^{2}+\left\|A^{r / 2} z\left(\vartheta_{t} \omega\right)\right\|_{2}^{2}+\left\|A^{(r+2) / 2} z\left(\vartheta_{t} \omega\right)\right\|^{2}+\left\|A^{r / 2} z\left(\vartheta_{t} \omega\right)\right\|^{2}+r_{0}^{2}(\omega), \\
& P=\max \left\{6, \frac{\left(1-\delta_{1}\right)^{2} C_{s}}{2 C_{U}^{2}}, k_{0}+4\right\}, \\
& \beta_{2}=\min \left\{\varepsilon-\frac{1}{2}-\frac{k_{0} \varepsilon}{4}, 2 D_{1}-2 \varepsilon-\frac{5}{2}-\frac{k_{0} D_{1}}{4}, \frac{3 k_{0}}{4}-2 \varepsilon-\frac{1}{2}, \delta-\frac{1+4 \varepsilon+4 \delta}{4 D_{1}}-k_{0}-\frac{5}{4}\right\}, \\
& \rho_{3}=\min \left\{\frac{2}{4-k_{0}}, D_{1}-\frac{5}{4}-\frac{k_{0} D_{1}}{8}, \frac{3}{8} k_{0}-\frac{1}{4}\right\} .
\end{aligned}
$$

(within the limited range of each parameter, each item in $\beta_{2}$ and $\rho_{3}$ is greater than zero).

For (4.21), using Gronwall's inequality

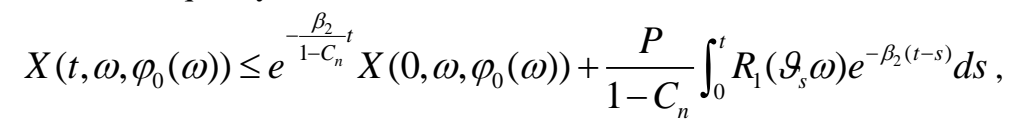

use $\vartheta_{-t} \omega$ instead of $\omega$ in the above formula to get

by Theorem 4.1,there is

$$
X\left(t, \vartheta_{-t} \omega, \varphi_{0}\left(\vartheta_{-t} \omega\right)\right) \leq e^{-\frac{\beta_{2}}{1-C_{n}} t} X\left(0, \vartheta_{-t} \omega, \varphi_{0}\left(\vartheta_{-t} \omega\right)\right)+\frac{P}{1-C_{n}} \int_{-t}^{0} R_{1}\left(\vartheta_{s} \omega\right) e^{\beta_{2} s} d s
$$

among there,

$$
\int_{-t}^{0} R_{0}\left(\vartheta_{s} \omega\right) e^{\beta_{2} s} d s \leq \int_{-t}^{0} \tilde{R}_{1}(s, \omega) e^{\beta_{2} s} d s \leq \int_{-\infty}^{0} \tilde{R}_{1}(s, \omega) e^{\beta_{2} s} d s<+\infty
$$

$$
\tilde{R}_{1}(s, \omega)=2\left(e^{\sigma|t|} r^{(1)}\left(A^{r / 2} \omega\right)\right)^{2}+\left(e^{\sigma|t|} r^{(1 / 2)}\left(A^{r / 2} \omega\right)\right)^{2}+\left(e^{\sigma|t|} r\left(A^{r / 2} \omega\right)\right)^{2}+r_{0}^{2}(\omega)
$$

Because of $\varphi_{0}\left(\vartheta_{-t} \omega\right) \in \bar{B}\left(\vartheta_{-t} \omega\right)$, so there is $T_{\bar{B}\left(r_{1}(\omega)\right)}>0$, so that for all $t \geq T_{\bar{B}\left(r_{1}(\omega)\right)}$, there is

So,

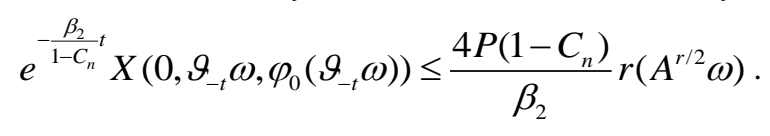

$$
\begin{aligned}
& X(t)=\left\|A^{r / 2} u_{N}\right\|_{2}^{2}+\left\|A^{r / 2} \omega_{N}\right\|^{2}+\left\|A^{r / 2} \theta_{N}\right\|^{2}+\left\|A^{r / 2} \eta_{N}^{t}\right\|_{M^{1}}^{2} \\
& \leq \frac{4 P\left(1-C_{n}\right)}{\beta_{2}} r\left(A^{r / 2} \omega\right)+\frac{P}{1-C_{n}} \int_{-\infty}^{0} \tilde{R}_{1}(s, \omega) e^{\beta_{2} s} d s=r_{1}^{2}(\omega) .
\end{aligned}
$$

To sum up, the theorem is proved.

Consider the space $\mathrm{M}^{1+r}$ corresponding to variable $\eta^{t}$, refer to [9], note that for any $t \geq 0, \omega \in \Omega$, there are

$$
\begin{gathered}
\eta_{N}^{t}\left(t, \vartheta_{-t} \omega, \varphi_{0}\left(\vartheta_{-t} \omega\right), s\right)=\left\{\begin{array}{c}
u_{N}\left(t, \vartheta_{-t} \omega, \varphi_{0}\left(\vartheta_{-t} \omega\right)\right)-u_{N}\left(t-s, \vartheta_{s-t} \omega, \varphi_{0}\left(\vartheta_{s-t} \omega\right)\right), s \leq t \\
\eta_{N}^{t}\left(t, \vartheta_{-t} \omega, \varphi_{0}\left(\vartheta_{-t} \omega\right)\right), s \geq t .
\end{array}\right. \\
\eta_{N s}^{t}\left(t, \vartheta_{-t} \omega, \varphi_{0}\left(\vartheta_{-t} \omega\right), s\right)=\left\{\begin{array}{c}
u_{N t}\left(t-s, \vartheta_{s-t} \omega, \varphi_{0}\left(\vartheta_{s-t} \omega\right)\right), s \leq t \\
0, s \geq t
\end{array}\right.
\end{gathered}
$$

Define collection $\tilde{B}(\omega)=\overline{\bigcup \varphi_{0}\left(\vartheta_{-t} \omega\right) \in \bar{B}\left(\vartheta_{-t} \omega\right) \bigcup_{t \geq 0} \eta_{N}^{t}\left(t, \vartheta_{-t} \omega, \varphi_{0}\left(\vartheta_{-t} \omega\right), s\right)}$, where $\varphi=\left(u, \omega, \theta, \eta^{t}\right)^{T}$ is the solution of equation (4.8). Then theorem 4.5, (4.22) and (4.23) can be obtained

$$
\max \left\{\left\|\eta_{N s}^{t}\left(t, \vartheta_{-t} \omega, \varphi_{0}\left(\vartheta_{-t} \omega\right), s\right)\right\|_{\mathrm{M}^{1+r}}^{2},\left\|\eta_{N}^{t}\left(t, \vartheta_{-t} \omega, \varphi_{0}\left(\vartheta_{-t} \omega\right), s\right)\right\|_{\mathrm{M}^{3+r}}^{2}\right\} \leq 2 r_{1}^{2}(\omega), \forall s \geq 0,
$$

so $\tilde{B}(\omega)$ in $\mathrm{M}^{r+1}=L_{\mu}^{2}\left(R^{+} ; v^{r+1}\right)$ is bounded.

Know by Theorem 4.3 and Theorem 4.5 


$$
\sup _{\eta^{t} \in \bar{B}(\omega), s \geq 0}\left\|\eta^{t}(s)\right\|_{2}^{2}=\sup _{t \geq 0} \sup _{\varphi_{0}\left(\vartheta_{-t} \omega\right) \in \bar{B}\left(\vartheta_{-t} \omega\right)}\left\|\eta_{N}^{t}\left(t, \vartheta_{-t} \omega, \varphi_{0}\left(\vartheta_{-t} \omega\right), s\right)\right\|_{2}^{2} \leq 2 r_{0}^{2}(\omega) .
$$

Therefore, for any $\eta^{t} \in \tilde{B}(\omega)$, there is

$$
\left\|\eta^{t}(s)\right\|_{\mathrm{M}^{1}}^{2}=\int_{0}^{\infty} \mu(s)\left\|\eta^{t}(s)\right\|_{2}^{2} d s \leq 2 r_{1}^{2}(\omega) \int_{0}^{\infty} \frac{1}{\sigma^{2}} e^{-\frac{s}{\sigma}} d s=\frac{2 r_{1}^{2}(\omega)}{\sigma} .
$$

That is, $\tilde{B}(\omega) \subset L_{\mu}^{2}\left(R^{+} ; H^{2}(U)\right) \cap H_{0}^{1}(U)$ is a bounded subset.

According to Theorem 2.2, $\tilde{B}(\omega)$ is tight in $L_{\mu}^{2}\left(R^{+} ; H^{2}(U)\right) \cap H_{0}^{1}(U)$.

The following proves that the random power system $\Phi(t, \omega)$ has a random attractor.

Theorem 4.6 Assuming conditions (1)-(4) hold, for any $\omega \in \Omega$, the random dynamic system $\Phi(t, \omega)$ generated by equation (4.8) has a compact random attractor set $\Lambda(\omega) \in V^{0}$, and has a random attractor $\mathrm{A}(\omega) \subseteq \Lambda(\omega) \bigcap B_{0}(\omega)$.
Proof: Any $\omega \in \Omega$, by theorem 4.5, let $B_{r}(\omega)=v^{r+2} \times v^{r} \times v^{r}$ be a random closed ball with a radius of $r_{1}(\omega)$, Definition $\Lambda(\omega)=B_{r}(\omega) \times \tilde{B}(\omega)$, then $\Lambda(\omega) \in D\left(V^{0}\right)$, because

$$
\begin{gathered}
v^{r+2} \times v^{r} \times v^{r} \rightarrow H_{0}^{2}(U) \times L^{2}(U) \times L^{2}(U) \quad \text { (Embed) } \\
B_{r}(\omega) \rightarrow H_{0}^{2}(U) \times L^{2}(U) \times L^{2}(U) \quad \text { (Embed) }
\end{gathered}
$$

using theorem 2.2 again, $\tilde{B}(\omega)$ is compact in $\mathrm{M}^{1}$, so $\Lambda(\omega)$ is compact in $V^{0}$. Next, prove the attractiveness of set $\Lambda(\omega)$.

For any $B(\omega) \in D\left(V^{0}\right)$

From Theorem 4.5

$$
\lim _{t \rightarrow \infty} d_{H}\left(\Phi\left(t, \vartheta_{-t} \omega\right) B\left(\vartheta_{-t} \omega\right), \Lambda(\omega)\right)=0
$$

Combined with Theorem 4.4 there is

$$
\varphi_{N}\left(0, \omega, \varphi_{0}(0, \omega)\right)=\varphi\left(0, \omega, \varphi_{0}(0, \omega)\right)-\varphi_{L}\left(0, \omega, \varphi_{0}(0, \omega)\right) \in \Lambda(\omega),
$$

$$
\mathrm{d}\left(\Phi\left(t, \vartheta_{-t} \omega\right) B\left(\vartheta_{-t} \omega\right), \Lambda(\omega)\right) \leq e^{-2 \beta_{1} t}\left(\left\|u_{0}\right\|_{2}^{2}+\left\|u_{1}+\varepsilon u_{0}\right\|^{2}+\left\|\theta_{0}\right\|^{2}+\left\|\eta_{0}\right\|_{M^{1}}^{2}\right) .
$$

Therefore, the random dynamic system $\Phi(t, \omega)$ generated by equation (4.8) has a random attractor $\mathrm{A}(\omega) \subseteq \Lambda(\omega) \cap B_{0}(\omega)$

To sum up, the theorem is proved.

\section{REFERENCES}

1. W.J,Ma,Q.Z.Ma,Attractors for Stochastic Strongly damped plate equations with additive noise,Electron.J.Differ.Equ.111(2013):1-12.

2. L.Aronld.random Dynamical Systems,SpringerVerlag, 1998

3. A.Pazy.Semigroups of Linear Operators and Applications to Partial Differential Equations,Appl.Math.Sci.vol.44,Springer-

Verlag,New York,1983.

4. M.H.Yang.J.Q.Duan,P.kloeden.Asymptotic behavior of solutions for random wave equation with nonlinear damping and white noise, Nonlinear Anal.12(2010):464-478

5. F. Zhou and M. Zhao, Random attractors for damped non-autonomous wave equations with memory and white noise, Nonlinear Anal. 120 (2015), 202-226

6. Shen Xiaoying,Ma Qiaozhen.The existences of random attractors for plate equations with memory and additive white noise, Korean $J$ .Math.2016,24:461-481.
7. Hao $\mathrm{Wu}$, Long-time behavior for a nonlinear plate equation with thermal memory.J.Math.Anal.Appl. 348 (2008) 650-670

8. Hans Crauel, Franco Flandoli, Attractors for random dynamical systems. Probab. Theory Relat. Fields 100, 365-393 Springer-Verlag (1994)

9. Hans Crauel, Arnaud Debussche, and Franco Flandoli, Random Attractors .Journal of Dynamics and DiJferential Equations, Vol. 9, No. 2, 1997

10. Z.W. Shen, S.F. Zhou, W.X. Shen, Onedimensional random attractor and rotation number of the stochastic damped sine-Gordon equation, J. Differential Equations 248(2010): 1432- 1457.

11. Shen Xiaoying,Ma Qiaozhen.Existences of random attractors for weakly dissipative plate equations with memory and additive white noise,Comput.Math.Appl.2017,73:2258-2271.

12. S. Woinowsky-Krieger, The effect of an axial force on the vibration of hinged bars, J. Appl. Mech. 17 (1950) 35-36.

13. H.M. Berger, A new approach to the analysis of large deflections of plates, J. Appl. Mech. 22 (1955) 465-472. 
14. Maurizio Grasselli,Jaime E.Munoz Rivera,Vittorino Pata,On the energy decay of the linear thermoelastic plate with memory.J.Math.Anal.Appl. 309 (2005) 1-14

15. V.Pata,A.Zucchi.Attractors for a damped hyperbolic equation with linear memory, Adv. Math. Sci.Appl.11(2001):505-529

16. M.E. Gurtin, A.C. Pipkin, A general theory of heat conduction with finite wave speeds, Arch. Ration. Mech. Anal. 31 (1968) 113-126.

17. S. Gatti, M. Grasselli, V. Pata, Exponential attractors for a conserved phase-field system with memory, Phys. D 189 (2004) 31-48.

18. C. Giorgi, M. Grasselli, V. Pata, Uniform attractors for a phase-field model with memory and quadratic nonlinearity, Indiana Univ. Math. J. 48 (1999) 1395-1445.
19. V. Belleri, V. Pata, Attractors for semilinear strongly damped wave equation on $R^{3}$,Discrete Contin.Dyn.Syst.(2001) 719-735

20. Liu Y , Ueda Y . Decay estimate and asymptotic profile for a plate equation with memory[J]. Journal of Differential Equations, 2019, 268(5).

21. Liu R, Chai S, Cao X . Exact controllability of thermoelastic plate equation with memory[J]. Mathematical Methods in the Applied Sciences, 2019.

22. Al-Mahdi A M . General stability result for a viscoelastic plate equation with past history and general kernel[J]. Journal of Mathematical Analysis and Applications, 2020.

23. Baowei Feng,Long-Time Dynamics of a Plate Equation with Memory and Time Delay.New Series

(2018)
49:395-418.

24. 